\title{
1 Jointing analysis of scATAC-seq datasets using epiConv
}

$3 \quad$ Li Lin ${ }^{1^{*}}$, Liye Zhang ${ }^{1^{*}}$

$4 \quad{ }^{1}$ School of Life Science and Technology, ShanghaiTech University, Shanghai, China.

$5 \quad{ }^{*}$ Correspondence should be addressed to L.L. (linli@shanghaitech.edu.cn) or L.Z.

6) (zhangly@shanghaitech.edu.cn).

7

\section{Abstract}

9 Technical improvement in ATAC-seq makes it possible to profile the chromatin states of single

cells at high throughput, but currently no method is available to integrate datasets from multiple

sources (different batches of same protocol or multiple experimental protocols). Here we present an algorithm to perform joint analyses on sCATAC-seq datasets from multiple sources. In addition to batch correction, we also demonstrate that epiConv is capable of aligning co-assay data 
19

\section{Text}

The expression of genes is regulated by transcription factors (TFs) that bind to the regulatory elements of the genome. As the accessible chromatin covers more than $90 \%$ TF binding regions, many techniques, such as Assay for Transposase-Accessible Chromatin using sequencing (ATACseq), have been developed to detect accessible chromatin ${ }^{1-3}$. Recent technical advancements have made it possible to profile the chromatin states of single cells at a high-throughput manner and along with other molecular modalities (e.g. transcriptome) in the same cells ${ }^{4-6}$. Similar to RNA-seq, ATAC-seq data also suffers from batch effects ${ }^{7}$. But batch correction on scATAC-seq data is more challenging as it is difficult to capture and correct batch derived variations on nearly binary chromatin profiles. For now, there are no specialized batch correction tool designed for scATAC-seq data to our knowledge. Most clustering algorithms for sCATAC-seq data capture biological information by learning a series of latent features from binary chromatin profiles. But whether latent features are confounded by batch effects and most importantly, whether batch effects can be removed by scaling these latent features by conventional ways used in scRNA-seq analysis, remain unclear.

Here, we develop a novel algorithm named epiConv (https://github.com/LiLinbiosoft/epiConv) for joint analysis of scATAC-seq data. EpiConv learns the similarities between cells by directly comparing their chromatin profiles (the dot product of two vectors of cells and followed by subsequent library size normalization) (Fig. 1a). Comparing to existing algorithms, epiConv performs similarly or slightly better on clustering and embedding (Fig. S1). However, its most significant advantage over other methods is that epiConv introduces an additional procedure to remove batch related bias in joint analysis of multiple datasets (Fig. 1b): it captures 
batch derived variations by Eigenvalue Decomposition and remove them by properly scaling the Eigen vectors (See method section for more details). Through comprehensive benchmarking, we demonstrated that epiConv was capable of removing batch effects and in the meantime retaining biological signals.

We first applied epiConv to a collection of peripheral blood mononuclear cells (PBMCs) datasets from two studies sequenced by two protocols (10x Genomics and dscATAC-seq) to benchmark its performance of batch correction ${ }^{5,8}$. For comparison, we also applied two commonly used clustering algorithms of scATAC-seq data (cisTopic ${ }^{9}$ and Latent Sematic Indexing ${ }^{4}$, LSI) to calculate a set of latent features and then removed the batch effects by scaling the latent features using Harmony ${ }^{10}$ (batch correction algorithm developed for scRNA-seq). Without batch correction, all three methods (epiConv, cisTopic and LSI) exhibited obvious batch effects between two studies (Fig. 1c). After batch correction, epiConv mixed single cells from two studies together, while cells were still partially or completely clustered by their data source in other methods (Fig. 1c). In order to quantitatively evaluate the performance of batch correction, we calculated the information entropy of batch mixing among each cell's nearest neighbors. EpiConv showed significant improvements of information entropy over other methods, which suggested that epiConv better mixed cells from multiple datasets together (Fig. 1d). To evaluate whether cells were grouped based on their biological identities, we performed differential analysis on single cells with known biological identities to get a set of marker chromatin regions for each known cell type. Given that CD8+ T cells grouped into two distinct clusters (naïve CD8+ cluster with accessible CCR7 promoter and effecter CD8+ cluster with accessible CCL5 promoter), we performed differential analysis to get markers for both naïve and effector CD8+ clusters. We 
summed the number of accessible peaks among these markers in single cells to calculate meta signatures that represented their similarities to known cell types. For each cell type, cells with high signature scores from two studies were grouped together and along with the corresponding cells with known identities (compare Fig. S2 with Fig1c), suggesting that epiConv retained biological heterogeneity.

EpiConv can also increase the resolution of chromatin profiles through joint analysis. Next, we demonstrated that aligning the ATAC-seq profiles of co-assay data (perform scRNA-seq and SCATAC-seq simultaneously on the same cell) onto sCATAC-seq references overcome the shortcomings of shallow sequencing in co-assay data and improve the performance of clustering and differentially accessible peaks calling. We integrated three datasets together (Fig. 2a), one co-assay dataset of mouse adult cerebral cortex ${ }^{6}$ (SNARE-seq), two scATAC-seq datasets of mouse adult whole brain ${ }^{5,11}$ (sci-ATAC-seq and dscATAC-seq) that served as references, whose sequencing depth are $\sim 5$ times deeper than the co-assay data. Single cells from three datasets were mixed together without obvious batch effects by epiConv batch correction (Fig. 2b), while other methods fail to do so (Fig. S3a). To assess whether the biological heterogeneity was retained after batch correction, we performed conventional clustering on co-assay dataset (Fig. 2a) and joint clustering on integrated dataset (Fig. 2d, clusters were manually annotated by canonical makers). More than $90 \%$ cells from each conventional cluster were assigned to only one joint cluster by epiConv and rare cell types (e.g. C03/Ex 6 and C11/Ex 7, see number of coassay cells in Fig. 2d) were also clearly segregated. On the contrary, other methods were less sensitive to rare cell types recognized by RNA-seq (Fig. S3b). We quantitatively evaluated the consistency of clustering by Adjusted Mutual Information (AMI), where higher AMI suggested 
higher consistency between conventional clustering and joint clustering after batch correction.

EpiConv showed the most consistent results among all methods ( 0.81 in Fig. 2 d; vs 0.66 and 0.63

in Fig. S3b). We also found that conventional cluster $\mathrm{C} 09$ could be further grouped into 3 cell

types by joint clustering (Fig. 2c, clusters highlighted by red circles), which referred to one major

cell type (oligodendrocyte) and two rare cell types (oligodendrocyte progenitor cell and

microglia) based on their expression profiles from co-assay data (Fig. 2d), suggesting that joint

analysis also revealed hidden rare cell types. Next, we examined whether single cells of co-assay

data were correctly assigned to the reference. In each dataset, we performed differential analysis

to get a set of marker peaks for each joint cluster to see whether these markers were conserved across different datasets. Although markers detected from two scATAC-seq references were consistent with each other, the results of co-assay data did not agree with them (Fig. S4a). Only markers of big clusters were conserved across three datasets (e.g. Ex 1-4). Moreover, a large number of cluster-specific markers could only be detected from deep sequencing references. We argued that this might be due to shallow sequencing of co-assay data and further incorporate RNA-seq profiles into our evaluation. To do that, we linked each peak to its closest gene promoter in the genome. Then for each cluster, we got a series of associated peaks with its cluster-specific marker genes (Fig. 2e). To quantitatively measure the consistency between ATAC-seq and RNAseq profiles, we calculated the fold change of enrichment for two types of markers ( $N_{\text {common }} /$ $\left(N_{A T A C} * N_{R N A}\right) * N_{\text {total; }} N_{\text {common }}$ n number of shared peaks, $N_{\text {ATAC, }}$ number of chromatin markers, $N_{R N A}$, number of marker genes associated peaks, $N_{\text {total }}$, number of total peaks). We expected that peaks residing near the promoter of one gene were more likely to be its regulators and clusterspecific chromatin markers would enrich in the nearby regions of marker genes if joint analysis 
correctly clustered recurrent cell types from co-assay and scATAC-seq datasets together. Indeed,

we observed the enrichment and for most clusters, much more chromatin markers could be

we found 2,719 differentially accessible peaks detected from the reference showed consistent pattern with the expressions of their nearest genes along the differentiation path that was

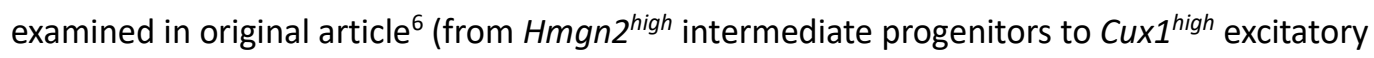
neurons, J01 to J04). Results based on both distinct cluster and continuous pseudotime 
143 lineage-specific regions. All malignant samples contained a large proportion of stem-like cells

144 (C01) but the composition of more differentiated cells varied among patients (Fig. S6b). The

147 normal cells (Fig. S6b). In contrast, malignant and normal cells were still clustered by their

148 biological conditions and patients in the results of cisTopic and LSI (Fig. S7a,b). In the original

149 article, Granja et al. ${ }^{12}$ developed a novel approach to project leukemic cells to normal 

the overlap of cluster-specific markers under normal and malignant hematopoiesis (e.g. malignant cells assigned to GMPs by Granja et al.'s method were actually more similar to monocytes). Through comparison of cluster-specific markers under normal and malignant hematopoiesis by epiConv, it could be seen that recalled peaks only explained a small fraction of heterogeneity of malignant cells, implying that leukemic cells might have alternative routes to

169 form hierarchy. Moreover, the alternative hierarchy of malignant cells might be easily missed by

170 other methods given that they could not deconvolute highly similar malignant cells into multiple

171 cell types (Fig. S7). Motivated by this thinking, we classified cluster-specific markers into three 
173 hierarchy loss (unique to normal hematopoiesis); 3) hierarchy gain (unique to malignant

174 hematopoiesis). The conserved hierarchies were controlled by the transcription factors that were

175 known to be important for hematopoiesis (e.g. RUNX in HSCs and MPPs, PU.1 in myeloid

176 lineages, E2A in lymphoid lineages, GATA in erythroid lineages). While the hierarchies unique to

177 normal hematopoiesis were controlled by the coordination of several epigenetic modifiers, they

178 were largely lost in malignant hematopoiesis (e.g CTCF lost in stem cells and erythroid lineages,

$179 \mathrm{C} / \mathrm{EBP}$ in myeloid lineages). These results highlighted the power of epiConv to learn the

180 heterogeneity of malignant cells through reference-guided analyses.

181 In this paper, we demonstrate the epiConv performed well in joint analysis of scATAC-seq data under various situations. Moreover, joint analysis provided deeper insights into the epigenetic regulation of single cells of different developmental stages as well as disease conditions. EpiConv is also computationally efficient and can be scaled to datasets with tens of thousands of cells

(Table S1). We believe that the computational framework in this study along with technical

190 Calculate the similarity matrix by epiConv EpiConv first calculated the similarity matrix $S$

191 between single cells from a binarized matrix $M$, where rows represent peaks and columns

192 represent cells. The similarity between two cells is calculated by the dot product of two cell

193 vectors, which means that the similarity matrix is calculated by $M^{T} M$. After normalization by 
description of this step can be found in Supplementary Note 1.

197 Correct batch effects by epiConv If single cells are from more than one batch, further steps are

required to remove the batch effects (Fig. 1b). We first apply Eigenvalue Decomposition to

normalized similarity matrix $S$ and keep $r$ Eigen values with largest absolute values and their

corresponding Eigen vectors $X$. The similarity matrix $S$ can be approximated by matrix product of

matrix $X$, which contains Eigen vectors and diagonal matrix $\Lambda$, whose diagonal elements contains

Eigen values. The difference between $S$ and its approximation is stored in residual matrix $S_{\text {residual. }}$

Eigen vectors $X$ captures batch derived variations and we assume that batch effects can be

corrected by properly scaling $X$ (Fig. S9a,b).

Next, we need to learn the recurrent cell populations across datasets. For simplicity, assume that

we have one reference dataset $A$ and one target dataset $B$ that needs be aligned to the reference

(Fig. S9c). For each cell in $B$, we find its $k_{1}$ nearest neighbors in $A$ based on the similarity matrix.

For each cell in $A$, we find its $k_{2}$ nearest neighbors in $B$. If one cell in $B$ (cell $b$ ) find a true neighbor

in $A$ (cell $a$ ) among its $k_{1}$ neighbors, we expect that cell $b$ should be similar with cell $a^{\prime} s k_{2}$

neighbors in $B$. To quantitatively measure the similarity between $b$ and $a^{\prime}$ s $k_{2}$ neighbors, we

require a collection of features of $B$ as guiding features (we will discuss later how to obtain these 
most of its initial $k_{1}$ neighbors. Cells in $B$ that have enough true neighbors in $A$ ( $>5$ in this study) are used as anchors for batch correction. For each anchor in $B$, we calculated the difference of Eigen vectors between itself and its true neighbors in $A$ as the scaling parameters. The scaling parameters for other non-anchor cells are calculated by the weighted mean of its nearest anchors (10 anchors in this study) on a shared nearest neighbors graph (SNN graph) of $B$, where the weight is equal to the edge of SNN graph. Finally, the corrected eigenvectors $X_{\text {corrected }}$ is calculated by scaling $X$ accordingly (Fig. S9b). and threshold $z$ control the number of anchors obtained. Smaller $k_{1}$ and threshold $z$ means more stringent filtering and fewer anchors. However, we expected that the number of anchors should be at least $\sim 20 \%$ of total cells for each dataset and distributed in all clusters (Fig. S9d). As we do not filter cells in $k_{2}$ neighbors, $k_{2}$ should be set to a smaller value compared to $k_{1}$ to prevent from picking too much false neighbors. In this study, we use $r=30, k_{1}=50, k_{2}=10$ unless otherwise noted. SCATAC-seq data, the Eigen vectors calculated above can be just used as guiding features given that Eigenvalue Decomposition also captures major biological variations. The SNN graph can be calculated based on the similarity matrix $S$ with $\mathrm{K}$-nearest neighbor set to $1 \%$ of total cells in $B$ 
SNN graph is calculated based on the information from RNA-seq or together with ATAC-seq, depending on the data quality of RNA-seq and ATAC-seq (see below for each co-assay dataset). Notably, the guiding features and SNN graph calculated by other methods are also compatible with epiConv if they better resolve the intra-population structure of the dataset.

EpiConv supports of multiple reference datasets and multiple target datasets. When multiple references and target datasets are available, we first integrate all references together (scale $\operatorname{Ref} B$ to match Ref $A$ by finding Ref $B$ 's neighbors in Ref $A$, then scale Ref $C$ to match $\operatorname{Ref} A$ and Ref $B$ by finding Ref $C$ 's neighbors in $\operatorname{Ref} A$ and $\operatorname{Ref} B$...) and then align each target dataset to the references by finding their neighbors in all references.

After the Eigen vectors of all datasets are properly scaled, the corrected similarity matrix is

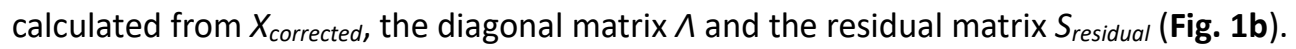

Dimension reduction and clustering of epiConv Given the similarity matrix $S$, we used Uniform and Louvain algorithm implemented in R package Seurat ${ }^{15}$ for clustering. In order to reduce noise, the similarity matrix between cells is first transformed to SNN graph $\left(S_{s n n}\right)$ described above. Then, the distance matrix $\left(\operatorname{Max}\left(S_{s n n}\right)-S_{s n n}\right)$ is used as UMAP input. The Louvain clustering is performed on the transformed UMAP graph $G$ from the distance matrix $\left(\operatorname{Max}\left(S_{s n n}\right)-S_{s n n}\right)$ to

257 generate consistent results between dimension reduction and clustering. All unmentioned adjusted between 0.2 and 0.8 . 
Detect differentially accessible peaks We use hypergeometric test to detect cluster-specific

accessible peaks. Population size was defined as total number of cells. Sample size was defined as

the sample was defined as number of cells with coverage in this cluster. Only peaks that were

considered as differentially accessible peaks. If peaks were considered as differentially accessible

peak to the cluster with lowest $p$-values.

Peak calling and matrix counting We used peaks defined by original article or called peaks from

ends of fragments by MACS2 ${ }^{16}$ (--nomodel --nolambda --keep-dup all --shift -95 --extsize 200) if

processed peak file was unavailable. We counted the ends of fragments against peaks to obtain the count matrix. The count matrix was binarized first for further analyses.

Application of other methods We applied cisTopic ${ }^{9}$ and Latent Sematic Indexing ${ }^{4}$ (LSI) to scATAC- 
mixing was calculated by the information entropy of batch among each cell's 200 nearest

neighbors after batch correction, where higher information entropy meant better mixing of cells

from different batches.

5,000 PBMCs data was processed as described above. In order to assess the performance of

epiConv and other methods on cell lines data ${ }^{3}$, we clustered cells into 8 clusters (the true number

of cell types) by louvain clustering and used Adjust Rand Index (ARI) to evaluate the accuracy.

scATAC-seq data of PBMCs We applied epiConv and other methods on two published PBMC

$T_{\text {cells }}^{8}$. The data from GSE123581 contains isolated CD4+ T cells, CD8+ T cells, CD19+ B cells, 
cortex from GSE126074 ${ }^{6}$ (SNARE-seq), scATAC-seq of mouse adult whole brain from Mouse Cell

Atlas $^{11}$ (sci-ATAC-seq) and GSE123581 ${ }^{5}$ (dscATAC-seq) together. For RNA-seq of co-assay data, we

used a common pipeline of Seurat with default settings (find 2,000 most variable genes and

obtain 50 PCs from them) to perform dimension reduction, clustering and finding cluster-specific

marker genes. Given that the sequencing depth of RNA-seq is deep but that of ATAC-seq is

analyses, we first aligned dscATAC-seq data to sciATAC-seq data and then aligned co-assay data to

them. The number of Eigen vectors ( $r$ ) was set to 40 and $k_{1}$ was set to 100 as the reference

datasets were large. Dimension reduction and joint clustering were performed on integrated 
of ATAC-seq to perform low dimensional embedding and clustering using the pipeline of Seurat.

The SNN graph used in batch correction was calculated on 100 combined features from RNA-seq and ATAC-seq. Clustering and differential analysis were performed as described above. Clusters of astrocytes (J12) and Hmgn high Intermediate progenitors (J01) were similar with each other and formed one cluster in unsupervised clustering. We performed further clustering to segregate them. Clusters J14, J15 and J16 were excluded in differential analysis as they did not contain coassay cells or were mixtures of rare cell types due to lack of proper reference cells. Cell types were labeled based on canonical markers. To investigate the changes of chromatin states and gene expression along differentiation, we also perform differential analyses on ATAC-seq and RNA-seq on 4 clusters (J01 to J04) and selected consistent chromatin markers and gene markers

337 (up-regulated in the same cluster). For cells sequenced by 10x Genomics from J01 to J04, we constructed their pseudotime axis by the sum of the number of accessible peaks among J04 markers minus that of $\mathrm{J} 01$ followed by library size normalization as we observed that from J01 to J04, most J01 markers gradually lost accessibility while J04 markers gained accessibility. Next, we

341 imputed the ATAC-seq counts from their 20 nearest neighbors from 10x Genomics data and

342 imputed the RNA-seq counts from their 20 nearest neighbors from SNARE-seq data. Finally, the

343 ATAC-seq counts and RNA-seq counts were normalized by library size with RNA-seq counts

344 further log2 transformed, and they were smoothed along the pseudotime axis by smooth.spline()

345 function in $\mathrm{R}$. 
and MPAL5 relapse) in Granja et al. $2019^{12}$. Although we could align them to normal

hematopoiesis, they share few common markers with normal cells compared to other samples.

351 We thought it was inappropriate to compare them with normal hematopoiesis and did not show

the results of these two samples. To test Granja et al.'s method, we first performed LSI on normal

cells with 25,000 differentially accessible peaks of lowest $p$-values, kept first 25 PCs, and

calculated the PCs of malignant cells using the parameters inferred normal cells. Then we applied

PCs of normal cells and then calculated the embedding of malignant cells by adding new unseen used the clusters from epiConv to annotate normal cells and the labels of malignant cells were assigned based on its nearest normal cell in the embedding of UMAP.

Motif calling was performed by Homer ${ }^{17}$ on the \pm 200 bp regions from peak centers. The length

Data availability All data analyzed in this article are available in public databases and are 
371

372

373

374

375

376

377

378

379

380

381

382

383

384

385

386

387

388

389

390

391

392

393

394

395

396

397

398

399

400

401

402

403

404

405

406

407

408

409

\section{References:}

1. Buenrostro, J.D., Giresi, P.G., Zaba, L.C., Chang, H.Y. \& Greenleaf, W.J. Transposition of native chromatin for fast and sensitive epigenomic profiling of open chromatin, DNAbinding proteins and nucleosome position. Nat Methods 10, 1213-1218 (2013).

2. Klemm, S.L., Shipony, Z. \& Greenleaf, W.J. Chromatin accessibility and the regulatory epigenome. Nat Rev Genet 20, 207-220 (2019).

3. Buenrostro, J.D. et al. Single-cell chromatin accessibility reveals principles of regulatory variation. Nature 523, 486-490 (2015).

4. Cusanovich, D.A. et al. Multiplex single cell profiling of chromatin accessibility by combinatorial cellular indexing. Science 348, 910-914 (2015).

5. Lareau, C.A. et al. Droplet-based combinatorial indexing for massive-scale single-cell chromatin accessibility. Nat Biotechno/37, 916-924 (2019).

6. Chen, S., Lake, B.B. \& Zhang, K. High-throughput sequencing of the transcriptome and chromatin accessibility in the same cell. Nat Biotechno/37, 1452-1457 (2019).

7. Orchard, P., Kyono, Y., Hensley, J., Kitzman, J.O. \& Parker, S.C.J. Quantification, Dynamic Visualization, and Validation of Bias in ATAC-Seq Data with ataqv. Ce/l Syst 10, 298-306 e294 (2020).

8. Satpathy, A.T. et al. Massively parallel single-cell chromatin landscapes of human immune cell development and intratumoral T cell exhaustion. Nat Biotechno/ 37, 925-936 (2019).

9. Bravo Gonzalez-Blas, C. et al. cisTopic: cis-regulatory topic modeling on single-cell ATAC-seq data. Nat Methods 16, 397-400 (2019).

10. Korsunsky, I. et al. Fast, sensitive and accurate integration of single-cell data with Harmony. Nat Methods 16, 1289-1296 (2019).

11. Cusanovich, D.A. et al. A Single-Cell Atlas of In Vivo Mammalian Chromatin Accessibility. Cel/ 174, 1309-1324 e1318 (2018).

12. Granja, J.M. et al. Single-cell multiomic analysis identifies regulatory programs in mixedphenotype acute leukemia. Nat Biotechno/37, 1458-1465 (2019).

13. Corces, M.R. et al. Lineage-specific and single-cell chromatin accessibility charts human hematopoiesis and leukemia evolution. Nat Genet 48, 1193-1203 (2016).

14. Mclnnes, L., Healy, J. \& Melville, J. UMAP: Uniform Manifold Approximation and Projection for Dimension Reduction. arXiv https://arxiv.org/abs/1802.03426v3 (2020).

15. Stuart, T. et al. Comprehensive Integration of Single-Cell Data. Ce//177, 1888-1902 e1821 (2019).

16. Zhang, Y. et al. Model-based analysis of ChIP-Seq (MACS). Genome Bio/9, R137 (2008).

17. Heinz, S. et al. Simple combinations of lineage-determining transcription factors prime cis-regulatory elements required for macrophage and B cell identities. Mol Ce// 38, 576589 (2010). 


\section{Acknowledgements}

411 This project was funded by the National Key Research and Development Program of China

412 (2018YFC1004602), National Natural Science Foundation of China (NSF 31871332) and a startup

413 fund to L.Z. from ShanghaiTech University. We would like to thank Vinay Kartha and Xiaoqi Zheng

414 for useful suggestions. We would like to thank Xiaojing Zhao for testing the reproducibility of the study. We would like to thank Yingdong Zhang on his technical support on the HPC platform of

416 ShanghaiTech University.

\section{Author contributions}

the manuscript. L.Z supervised the study.

\section{Competing interests}

423 The authors declare no competing interests. 
(a) Count matrix $M$

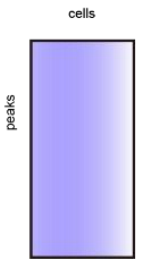

(b)

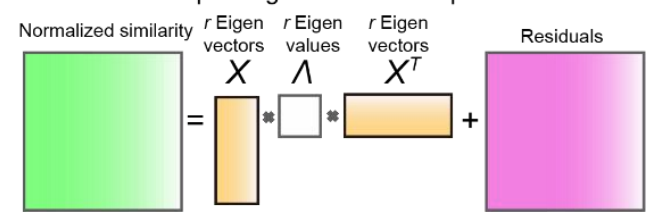

Step 2: correct Eigen vectors

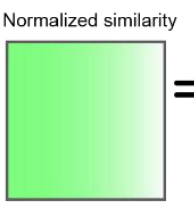
KNN graph

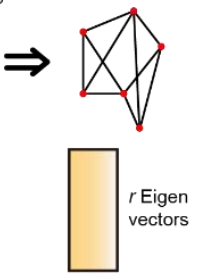

(c)

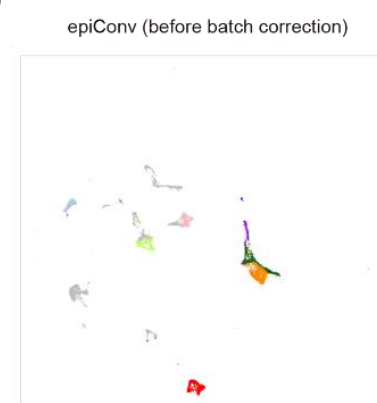

epiConv (after batch correction)

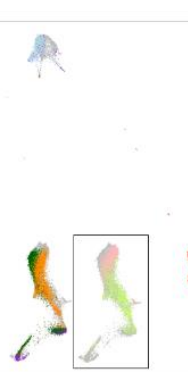

Pairwise similarity

cells

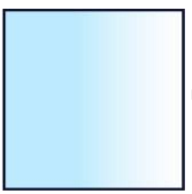

normalization

$\Rightarrow$

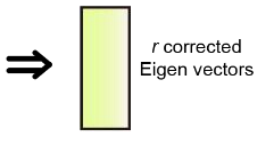

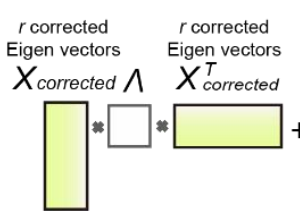
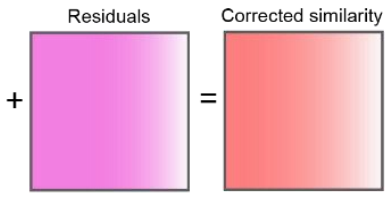

Corrected similarity

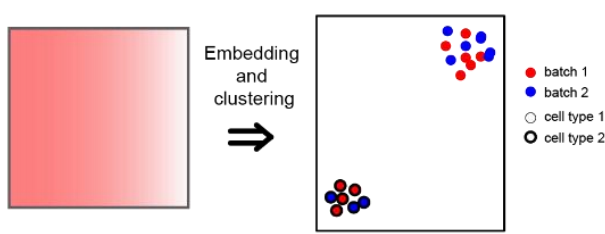

LSI

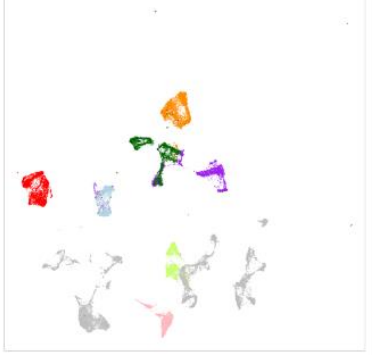

LSI (Harmony correction)

(d) Entropy of mixing

cisTopic (Harmony correction)
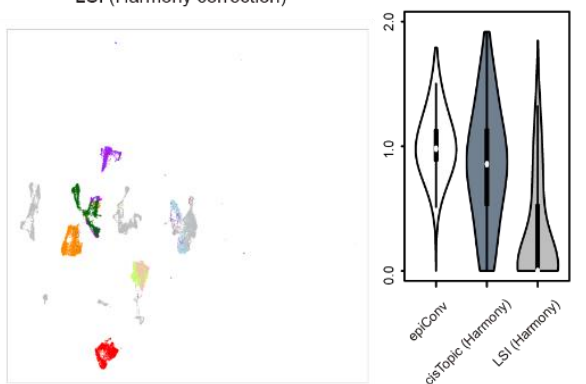

Figure 1 An overview of epiConv algorithm. (a) The workflow of calculating similarity matrix between single cells. (b) The workflow of batch correction. (c) epiConv better corrects batch effects than other methods on a collection of PBMCs datasets. (d) The entropy of batch mixing among cells' nearest neighbors after batch correction of Figure $1 \mathrm{c}$. 
(a)

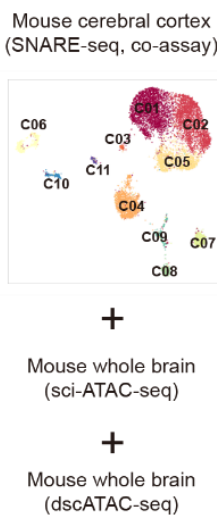

(b)

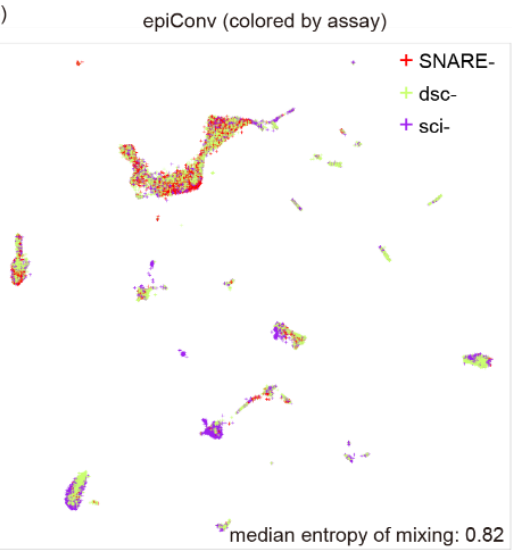

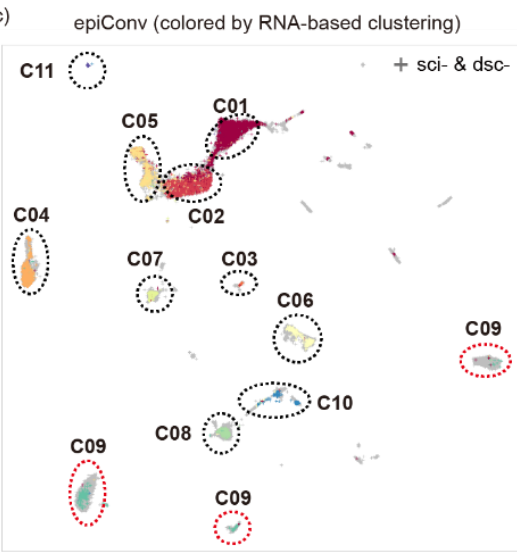

(d)

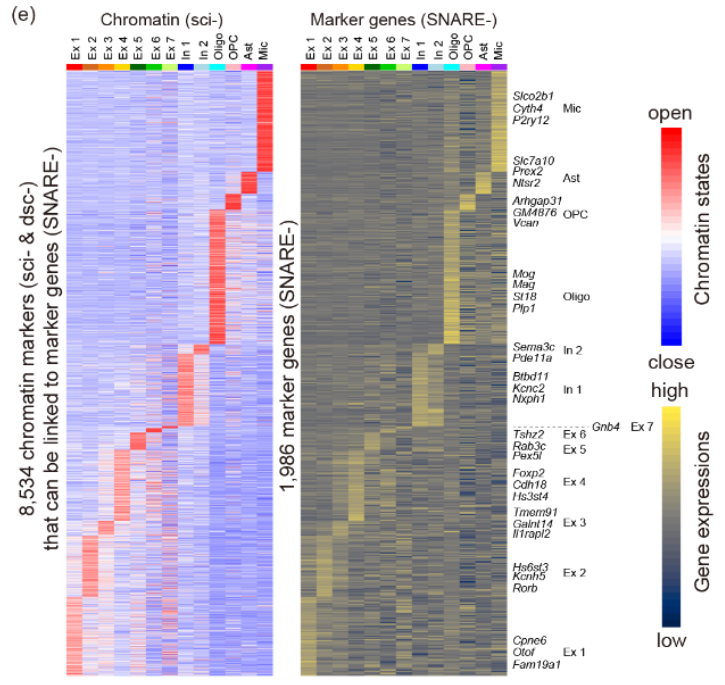

Figure 2 Aligning co-assay (SNARE-seq) data of mouse cerebral cortex to scATAC-seq (sci-ATAC-seq and dscATAC-seq) references increases the resolution of chromatin profiles. (a) Low dimensional embedding and clustering of co-assay data. (b) Joint embedding of co-assay data and scATAC-seq references, cells are colored by assays. Median value of entropy of batch mixing is shown in bottom right. (c) Joint embedding of co-assay data and scATAC-seq references, cells of co-assay data are colored according to conventional clustering in (a). Cells of sCATAC-seq references are colored in grey. (d) Joint embedding of co-assay data and scATAC-seq references, cells are colored by manually annotated joint clusters. Ex, excitatory neuron; In, inhibitory neuron; Oligo, oligodendrocyte; OPC, oligodendrocyte progenitor cell; Ast, astrocyte; Mic, microglia. (e) Left: heatmap of chromatin markers detected from scATAC-seq references that can be linked to marker genes. Right: expressions of the corresponding marker genes detected from RNA-seq of co-assay data. Selected marker genes with highest fold changes are shown in the right panel. 
(a)

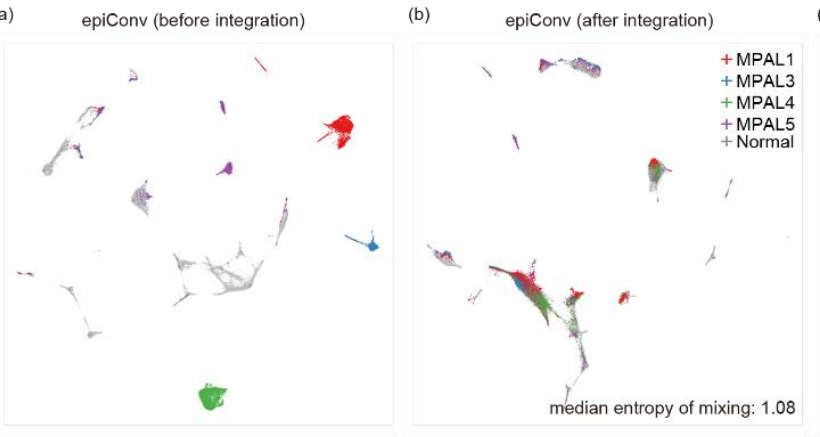

(c)

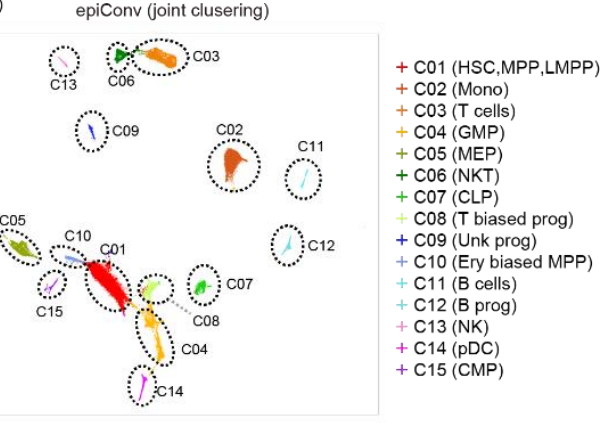

(d) Granja et al. 2019,

project to existing embedding

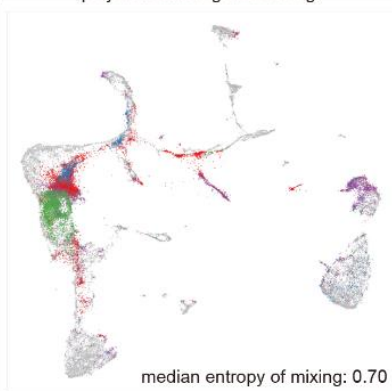

(e) Granja et al. 2019 (clusters assigned)

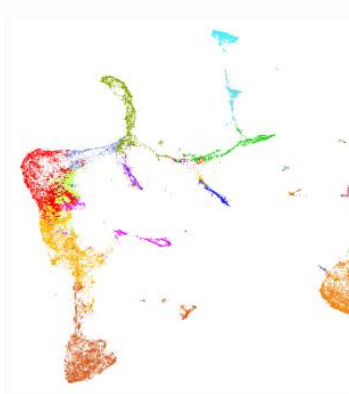

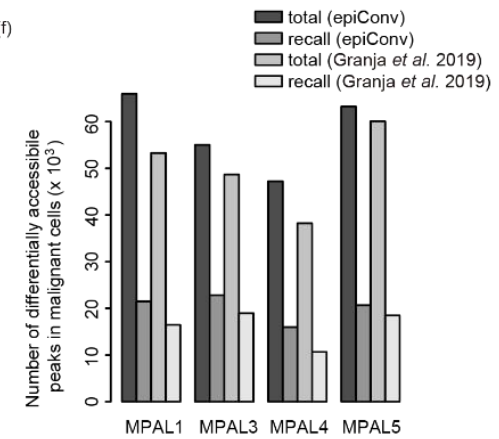

447

448

449

450

451

452

453

454

455

456

457

458

459

460

461

Figure 3 Aligning malignant cells to normal hematopoiesis reveals the hidden epigenetic hierarchy of leukemia. (a) Low dimensional embedding of epiConv before integration. (b) Low dimensional embedding of epiConv after integration. (c) Joint clustering of normal and malignant cells by epiConv. HSC, hematopoietic stem cell; MPP, multipotent progenitors; LMPP lymphoidprimed multipotent progenitor; Mono, monocyte; GMP, granulocyte-macrophage progenitor; MEP, megakaryocyte-erythroid progenitor; CLP, common lymphoid progenitor; Ery biased MPP, erythroid biased MPP; pDC, plasmacytoid dendritic cell; CMP, common myeloid progenitor. (d) Low dimensional embedding of Granja et al.'s method by projecting malignant cells to existing embedding of normal cells. Cells in (a), (b) and (d) are colored by patients. Median values of entropy of batch mixing in (b) and (d) are shown in bottom right. (e) Cluster assignments by Granja et al.'s method. Clusters of normal cells are identical as (c) and clusters of malignant cells are assigned based on their nearest normal cells. (f) Number of differentially accessible peaks in total and number of recalled differentially accessible peaks under normal and malignant hematopoiesis. 


\section{Supplementary Materials}


(a)

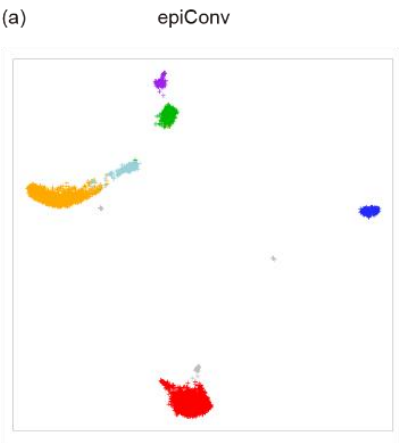

(b)

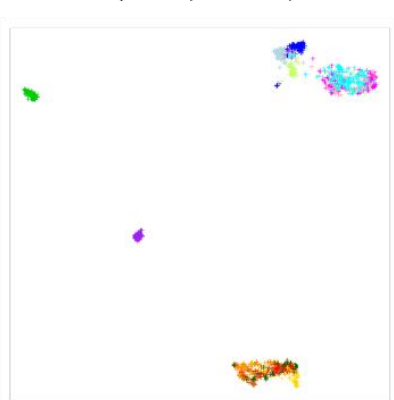

cisTopic

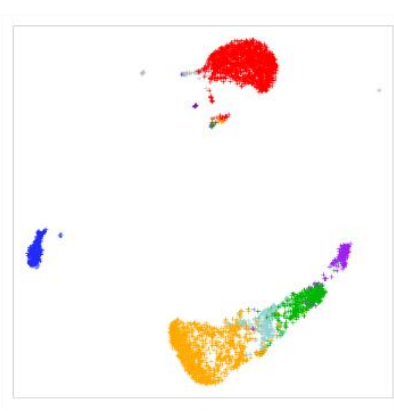

cisTopic $(A R I=0.47)$

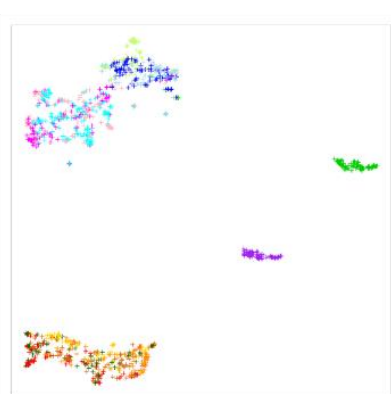

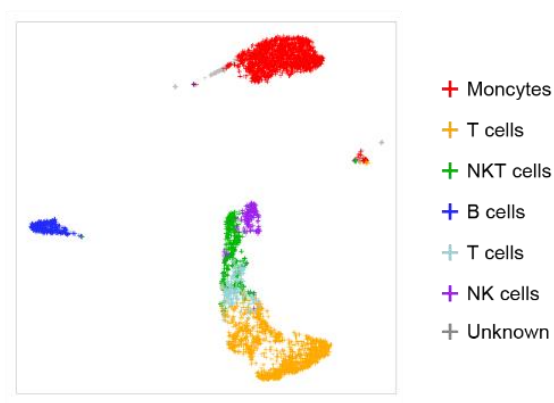

$\operatorname{LSI}(A R I=0.45$

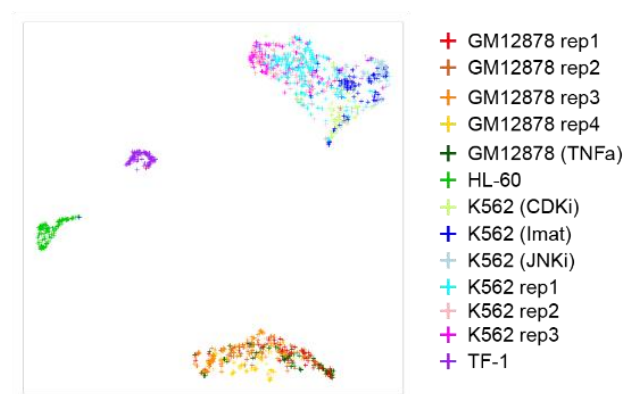

Figure S1 Comparison of epiConv with cisTopic and LSI. (a) Low-dimensional embedding of 5,000 PBMCs. Clusters are inferred from epiConv and manually annotated. Results from three methods are largely consistent. (b) Low-dimensional embedding of cell lines data. Cells are colored by their identities. Clustering of epiConv achieves higher Adjusted Rand Index (ARI) as it better segregates K562 cells treated by different drugs from untreated cells. 
(a)

GSE129785

B cells signature

Monocytes signature

a.

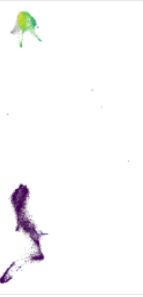

NK cells signature

Q

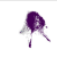

8
$\mathrm{CD} 4+\mathrm{T}$ cells signature

A
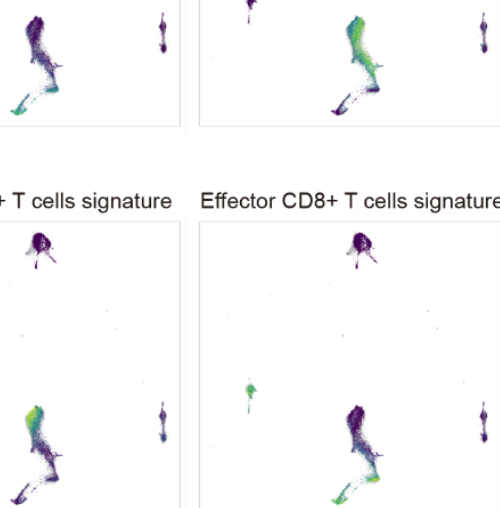

open

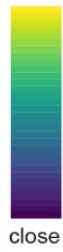

close

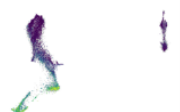

(b)

GSE123581

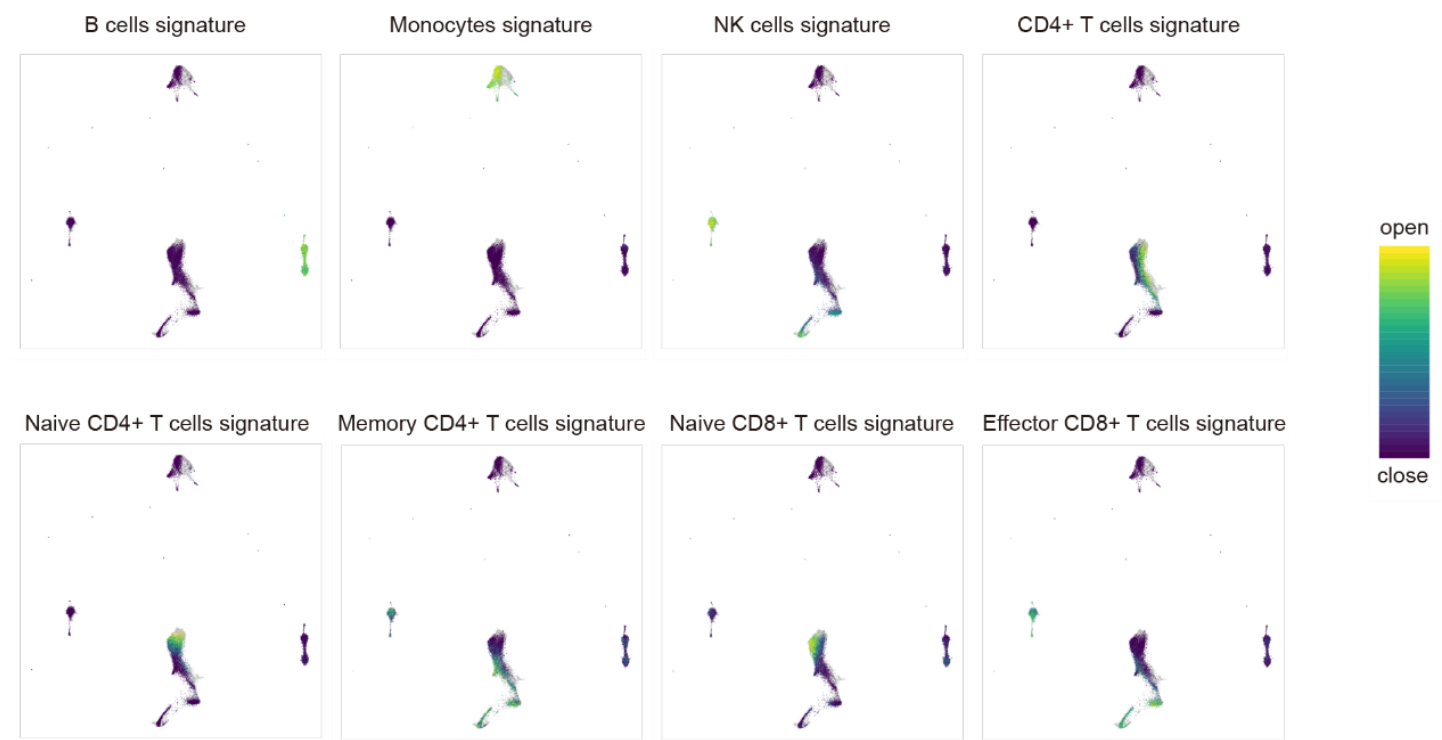

471

472

473

474
Figure S2 Supplementary figures for Fig. 1c. (a) Cell type specific signatures of PBMCs from GSE129785. (b) Cell type specific signatures of PBMCs from GSE123581. 
(a)

$$
\text { cisTopic }
$$

cisTopic (Harmony correction)

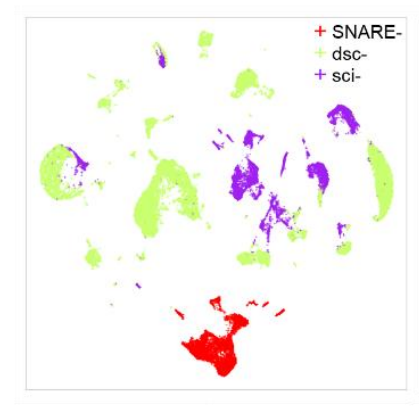

LSI

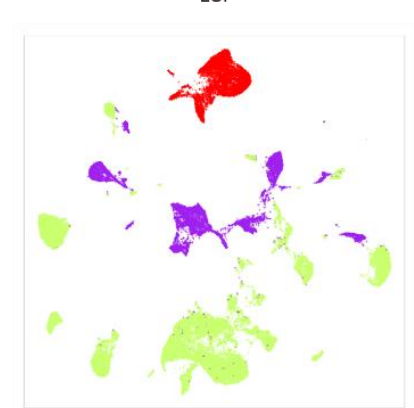

LSI (Harmony correction)

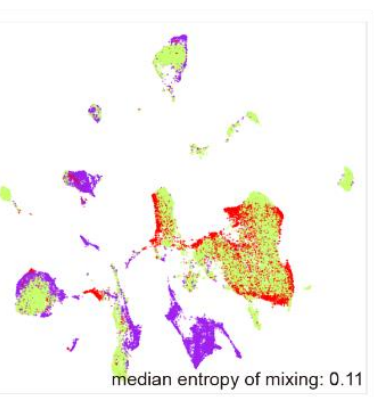

(b)

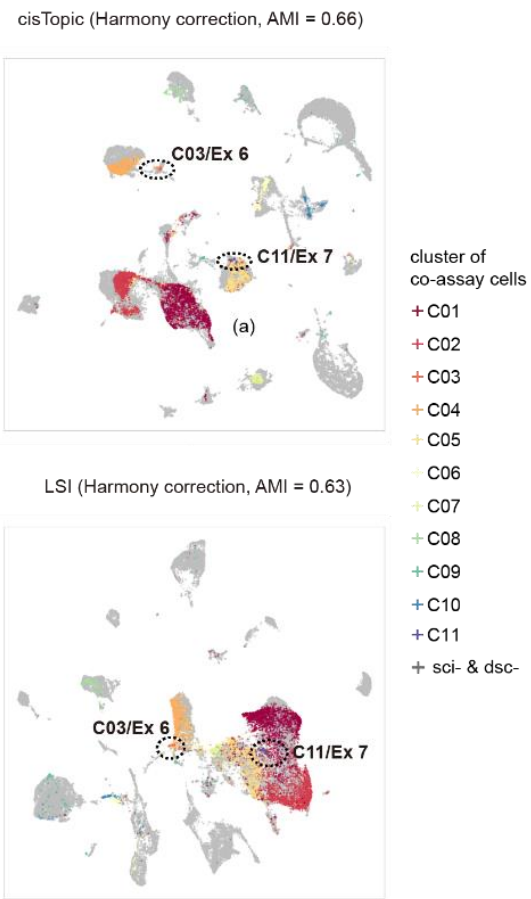

Figure S3 Benchmarking of cisTopic and LSI on adult mouse brain data. (a) Low-dimensional embedding of cisTopic and LSI before and after Harmony correction. Cells are colored by assays. Median values of entropy of batch mixing after batch correction are shown in bottom right. (b) Low-dimensional embedding of cisTopic and LSI after Harmony correction. Cells are colored according to conventional clustering of co-assay single cells in Fig. 2a. Cells of scATAC-seq references are colored in grey. Rare cell types of co-assay data (C03/Ex 6 and C11/Ex 7) are highlighted by dashed circles. 
bioRxiv preprint doi: https://doi.org/10.1101/2020.02.13.947242; this version posted January 3, 2021. The copyright holder for this preprint (which was not certified by peer review) is the author/funder, who has granted bioRxiv a license to display the preprint in perpetuity. It is made available under aCC-BY-NC-ND 4.0 International license.

(a)
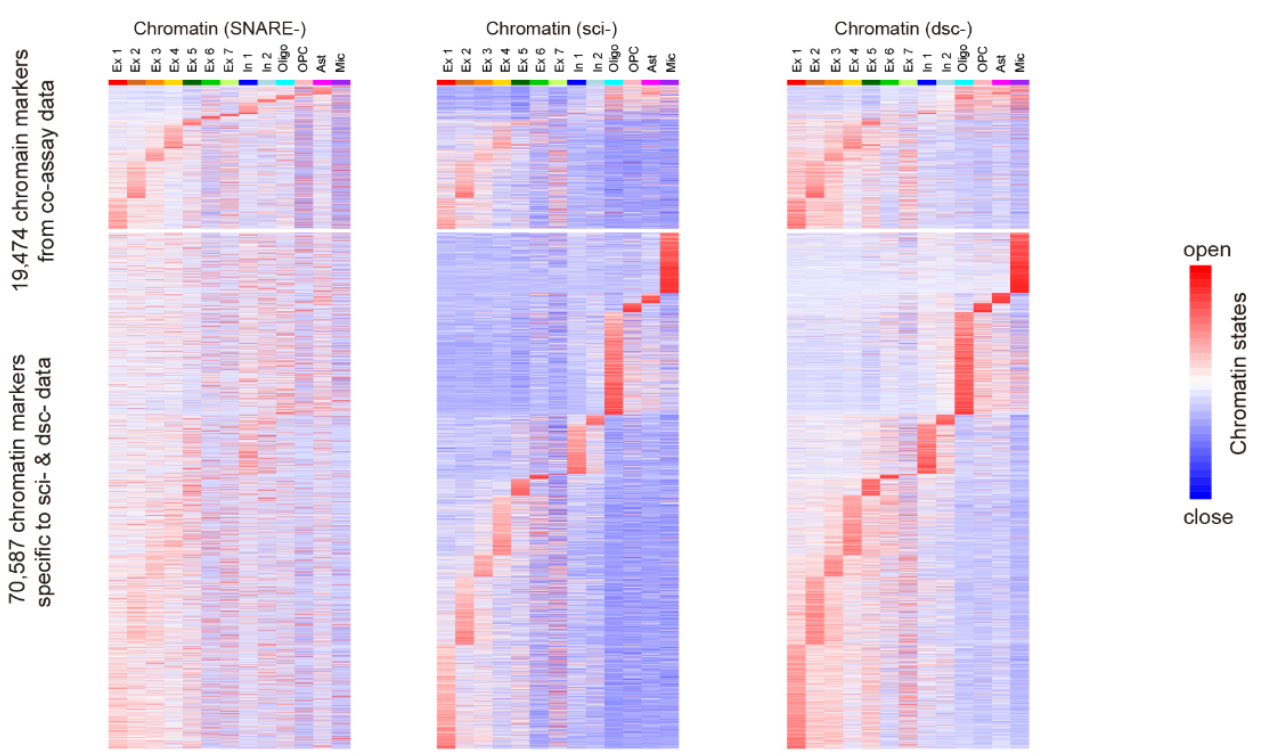

(b)
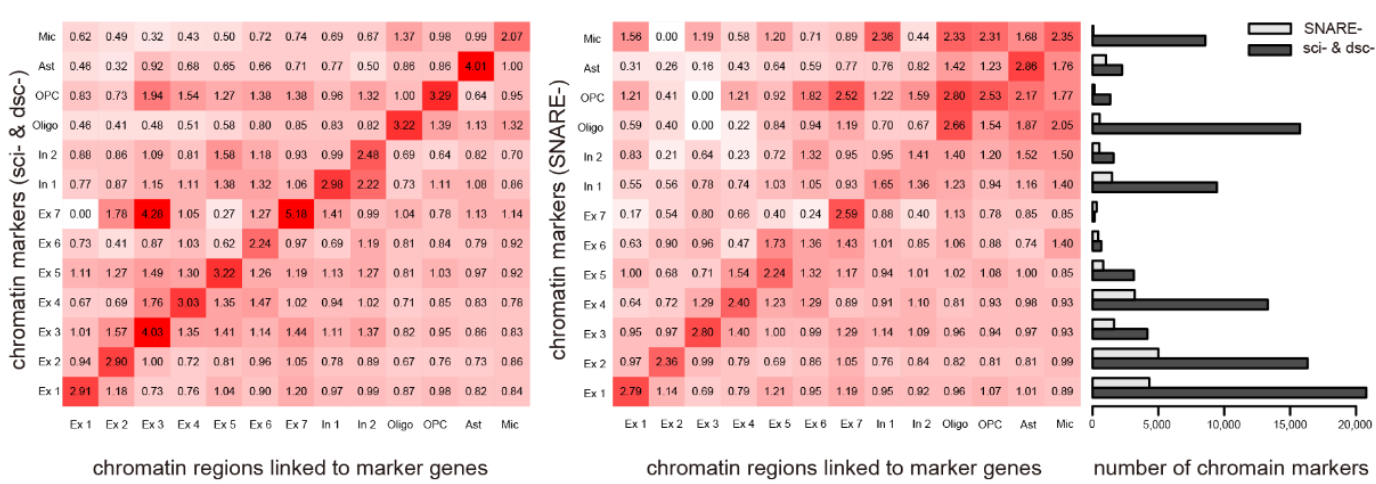

(c)

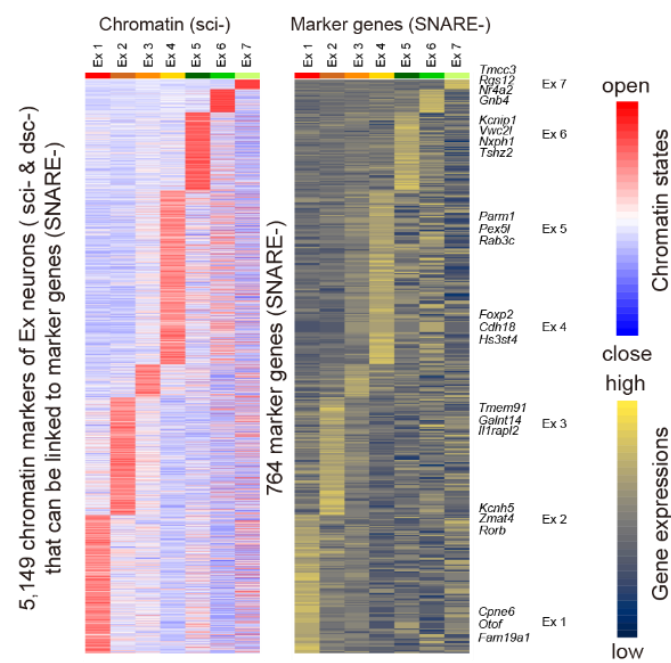

(d)

number of chromain markers

Figure S4 supplementary figures for Fig. 2. (a) Heatmaps of cluster-specific marker regions detected from ATAC-seq of co-assay data and two scATAC-seq references. Each column represents one aggregated ATAC-seq profiles of cells from corresponding joint clusters. (b) Left (scATAC-seq) and middle (co-assay): The fold changes of enrichment between ATAC-seq defined marker regions and RNA-seq defined associated regions. Right: the number of detected markers from scATAC-seq 
bioRxiv preprint doi: https://doi org/10.1101/2020.02 13.947242; this version posted January 3, 2021. The copyright holder for this preprint (which was not certified by peer review) is the author/funder, who has granted bioRxiv a license to display the preprint in perpetuity. It is made available under aCC-BY-NC-ND 4.0 International license.

490 references and co-assay data for each cluster. (c) Heatmaps of chromatin marker regions of

491 excitatory neurons detected from scATAC-seq references (left) and the expressions of

492 corresponding marker genes (right). Selected marker genes with highest fold changes are shown

493 in the right panel. (d) The fold changes of enrichment between ATAC-seq defined marker regions

494 and RNA-seq defined associated regions among excitatory neurons.

495 
bioRxiv preprint doi: https://doi.org/10.1101/2020.02.13.947242; this version posted January 3, 2021. The copyright holder for this preprint (which was not certified by peer review) is the author/funder, who has granted bioRxiv a license to display the preprint in perpetuity. It is made available under aCC-BY-NC-ND 4.0 International license.
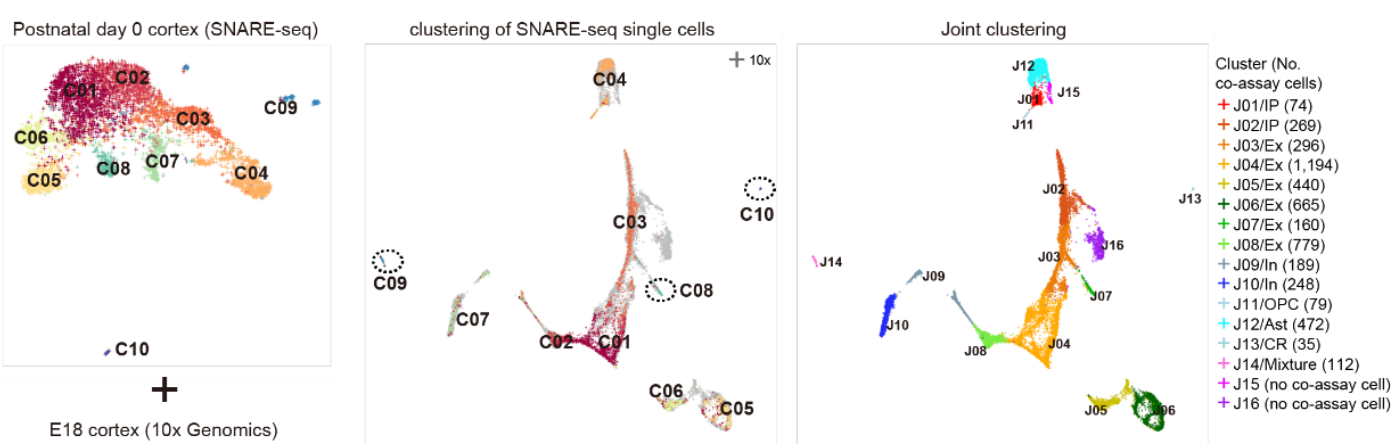

(b)

Chromatin (SNARE-)

Chromatin (10x)

(c)
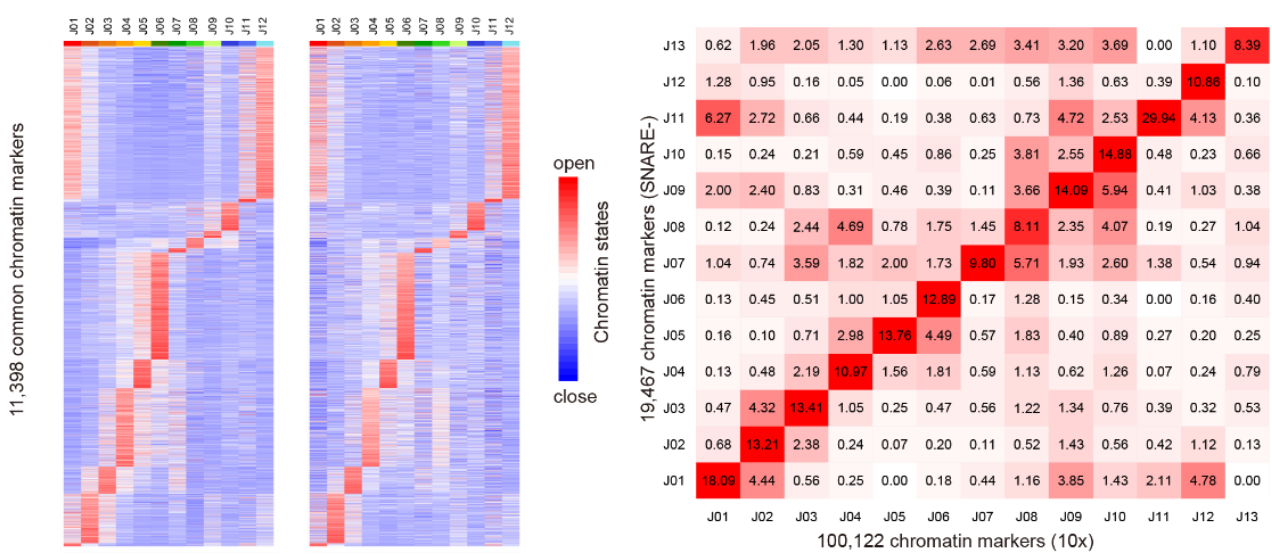

(d)
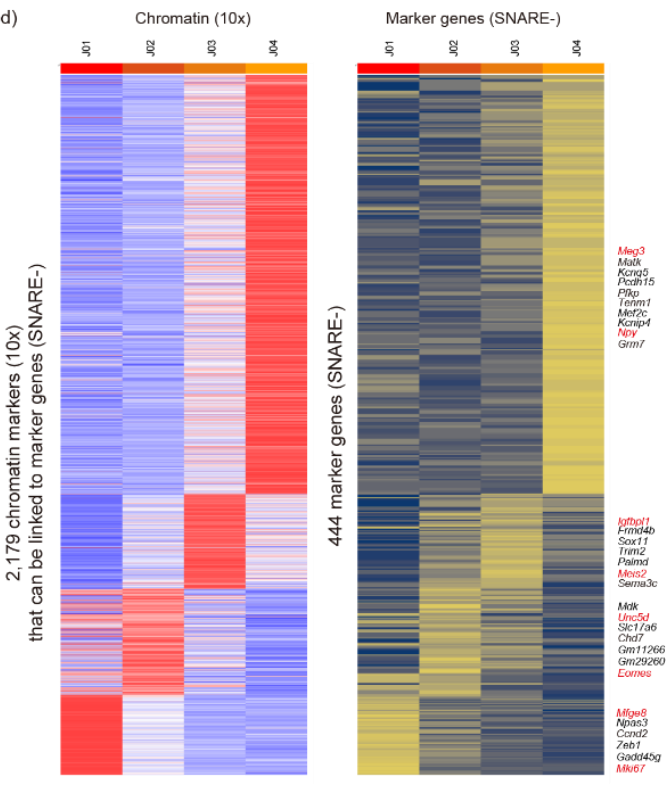

(e)

Figure S5 Aligning co-assay (SNARE-seq) data of postnatal day 0 mouse cortex to scATAC-seq (10x Genomics) reference. (a) Left: low dimensional embedding and conventional clustering of coassay data. Middle: joint embedding of co-assay data and scATAC-seq reference, cells of co-assay data are colored according to conventional clustering in the left. Right: joint embedding of coassay data and SCATAC-seq data, cells are colored according to annotated joint clusters. IP, intermediate progenitor; CR, Cajal-retzius cell. (b) Heatmaps of cluster-specific chromatin markers shared by two datasets. Each column represents one aggregated ATAC-seq profiles of joint clusters. (c) The fold changes of enrichment between chromatin markers detected from 

made available under aCC-BY-NC-ND 4.0 International license.

505 ATAC-seq of co-assay data and scATAC-seq reference. (d) Changes of chromatin states and gene 506 expressions from intermediate progenitors to excitatory neurons (J01-J04). Left: heatmap of 507 chromatin markers detected from SCATAC-seq reference that can be linked to marker genes.

508 Right: expressions of the corresponding marker genes. Selected marker genes with highest fold 509 changes are shown in the right panel. (e) Smoothed chromatin and expression profiles of 510 selected makers along the pseudotime of differentiation. 
bioRxiv preprint doi: https://doi.org/10.1101/2020.02.13.947242; this version posted January 3, 2021. The copyright holder for this preprint (which was not certified by peer review) is the author/funder, who has granted bioRxiv a license to display the preprint in perpetuity. It is made available under aCC-BY-NC-ND 4.0 International license.

(a)

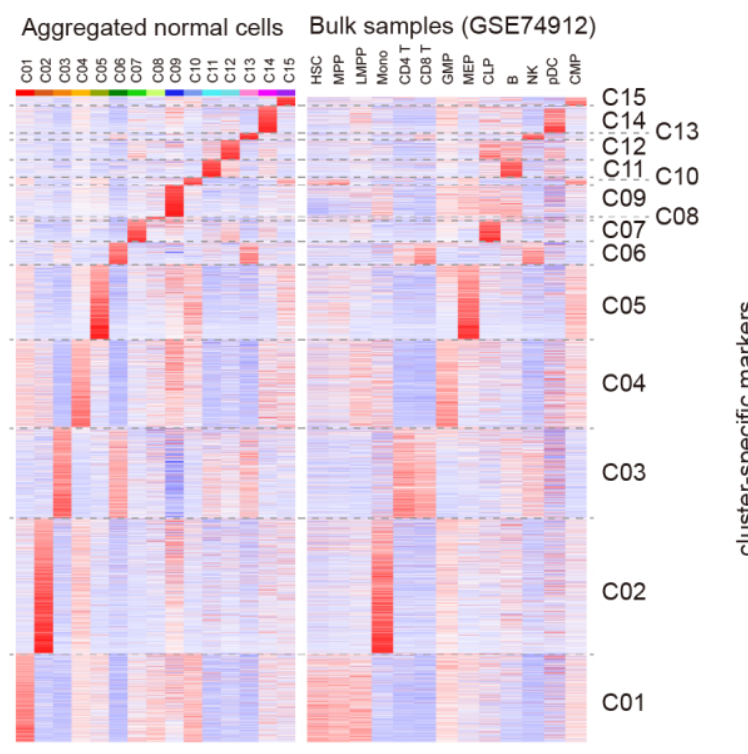

(b)

$\begin{array}{lllllllllllllll}\text { C15 } & 2.26 & 0.87 & 0.24 & 1.68 & 4.88 & 0.59 & 0.75 & 0.89 & 0.69 & 12.92 & 0.59 & 10.18\end{array}$ \begin{tabular}{lllllll|l|l|l|l|l|l} 
C14 & 3.57 & 0.84 & 0.18 & 2.85 & 0.84 & 0.65 & 4.73 & 1.08 & 1.97 & 0.90 & 2.32 & 0.65
\end{tabular} $\begin{array}{lllllllllllllll}\text { C13 } & 1.84 & 1.37 & 1.05 & 1.42 & 0.96 & 9.17 & 1.23 & 2.39 & 1.00 & 1.03 & 1.33 & 3.28\end{array}$ (2) C C C $\begin{array}{lllllllllllllll}2.75 & 1.14 & 0.76 & 1.96 & 0.82 & 1.02 & 2.29 & 3.82 & 2.73 & 0.70 & 10.23 & 1.51\end{array}$ $\begin{array}{llllllllllllll}\overline{\bar{\delta}} & C 11 & 1.48 & 1.78 & 0.72 & 0.99 & 0.67 & 1.28 & 1.03 & 1.12 & 0.92 & 1.04 & 34.99 & 2.45\end{array}$ $\begin{array}{lllllllllllllll}\overline{\text { त }} & \mathrm{C} 10 & 3.05 & 0.45 & 0.43 & 1.19 & 3.50 & 0.10 & 1.18 & 1.59 & 0.66 & 14.64 & 0.24 & 1.71\end{array}$ $\begin{array}{lllllllllllllllll}\mathrm{C} 09 & 0.64 & 0.28 & 0.16 & 3.79 & 0.53 & 0.06 & 0.95 & 0.73 & 15.42 & 0.27 & 0.19 & 0.19\end{array}$ $\begin{array}{lllllllllllllll}\cos & 0.78 & 0.51 & 2.70 & 1.72 & 0.36 & 2.42 & 0.80 & 16.62 & 3.85 & 0.00 & 2.43 & 3.01\end{array}$ $\begin{array}{lllllllllllllll}\text { C07 } & 3.50 & 0.87 & 0.54 & 2.64 & 0.42 & 0.56 & 5.28 & 2.63 & 2.73 & 0.32 & 2.87 & 0.43\end{array}$ $\begin{array}{llllllllllllllll}\operatorname{co6} & 1.49 & 1.30 & 1.59 & 0.79 & 0.46 & 20.49 & 1.73 & 2.98 & 0.52 & 0.83 & 1.77 & 3.90\end{array}$ $\begin{array}{llllllllllllllll}\operatorname{Co5} & 2.17 & 0.38 & 0.22 & 1.22 & 12.44 & 0.35 & 1.07 & 1.34 & 1.65 & 6.08 & 0.36 & 1.35\end{array}$

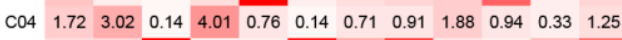
\begin{tabular}{lllllll|l|l|l|l|l|l|l}
$\mathrm{C} 03$ & 1.02 & 1.40 & 14.82 & 0.58 & 0.40 & 8.95 & 2.92 & 7.36 & 0.78 & 0.54 & 2.38 & 5.46
\end{tabular} $\begin{array}{llllllllllllllll}\mathrm{C} 02 & 0.27 & 6.30 & 0.24 & 1.32 & 0.47 & 0.48 & 0.28 & 0.66 & 1.11 & 0.51 & 1.34 & 2.73\end{array}$ $\begin{array}{lllllllllllll}\text { C01 } & 5.37 & 0.96 & 0.29 & 2.41 & 0.78 & 0.30 & 2.43 & 1.87 & 1.23 & 1.50 & 0.49 & 0.70\end{array}$ $\begin{array}{lllllllllllll}\text { Clusters } & \mathrm{C} 01 & \mathrm{C0} 2 & \mathrm{C} 03 & \mathrm{C} 04 & \mathrm{C} 05 & \mathrm{C} 06 & \mathrm{C} 07 & \mathrm{C} 08 & \mathrm{C} 09 & \mathrm{C} 10 & \mathrm{C} 11 & \mathrm{C} 15\end{array}$

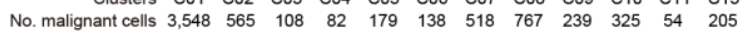

\section{Clusters of MPAL 1}

$\begin{array}{lllllllllll}\text { C15 } & 1.03 & 2.23 & 0.26 & 2.33 & 0.80 & 0.83 & 1.27 & 11.75 & 0.55\end{array}$ \begin{tabular}{l|l|lllllllll} 
C14 & 4.61 & 0.27 & 0.26 & 0.66 & 2.36 & 0.36 & 1.25 & 0.40 & 18.70
\end{tabular} $\begin{array}{llllllllllll}\text { C13 } & 1.30 & 0.92 & 0.72 & 1.77 & 0.86 & 2.69 & 1.91 & 1.16 & 2.33\end{array}$ \begin{tabular}{lllll|l|l|l|l|l}
$\mathrm{C} 12$ & 2.10 & 0.38 & 0.37 & 0.58 & 7.04 & 5.49 & 2.23 & 1.00 & 6.23
\end{tabular}

을 $\begin{array}{llllllllllll}\mathrm{C} & \mathrm{C} 11 & 1.49 & 0.82 & 1.76 & 0.92 & 5.13 & 1.76 & 1.57 & 1.11 & 6.44\end{array}$

$\begin{array}{lllllllllllll}\overline{\overline{\mathcal{O}}} & \mathrm{C} 10 & 1.50 & 0.66 & 0.27 & 1.23 & 1.16 & 0.69 & 1.46 & 13.78 & 0.59\end{array}$

$\begin{array}{llllllllllll}\overline{\text { ब }} & \operatorname{cog} & 0.45 & 0.27 & 0.11 & 0.89 & 1.23 & 0.54 & 11.66 & 0.21 & 0.65\end{array}$ $\begin{array}{lllllllllll}\cos & 0.35 & 0.00 & 0.36 & 0.00 & 0.33 & 22.08 & 2.32 & 0.78 & 0.57\end{array}$ \begin{tabular}{ll|l|l|l|l|l|l|l|l|l}
$C 07$ & 4.21 & 0.21 & 0.33 & 0.34 & 17.01 & 2.51 & 2.19 & 0.36 & 2.03
\end{tabular} $\begin{array}{lllllllllllll}\operatorname{Co6} & 1.58 & 0.81 & 3.39 & 1.14 & 1.46 & 4.01 & 0.77 & 0.80 & 1.81\end{array}$ $\begin{array}{llllllllllll}\operatorname{Co5} & 1.39 & 0.73 & 0.32 & 1.44 & 1.00 & 1.29 & 2.03 & 5.83 & 0.80\end{array}$ $\begin{array}{llllllllllll}\mathrm{C} 04 & 1.56 & 3.27 & 0.06 & 3.92 & 1.07 & 0.89 & 1.78 & 1.69 & 0.98\end{array}$ $\begin{array}{lllllllllll}\mathrm{C} 03 & 0.90 & 0.27 & 14.33 & 0.44 & 1.13 & 6.90 & 0.55 & 0.83 & 1.20\end{array}$ $\begin{array}{llllllllllll}\mathrm{C} 02 & 0.35 & 5.56 & 0.34 & 2.66 & 0.73 & 1.40 & 1.57 & 1.04 & 1.10\end{array}$ $\begin{array}{llllllllllll}\mathrm{C} 01 & 6.52 & 0.74 & 0.29 & 1.36 & 1.42 & 1.68 & 1.54 & 3.04 & 0.74\end{array}$

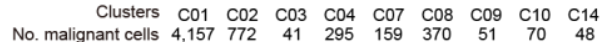

$$
\text { Clusters of MPAL4 }
$$

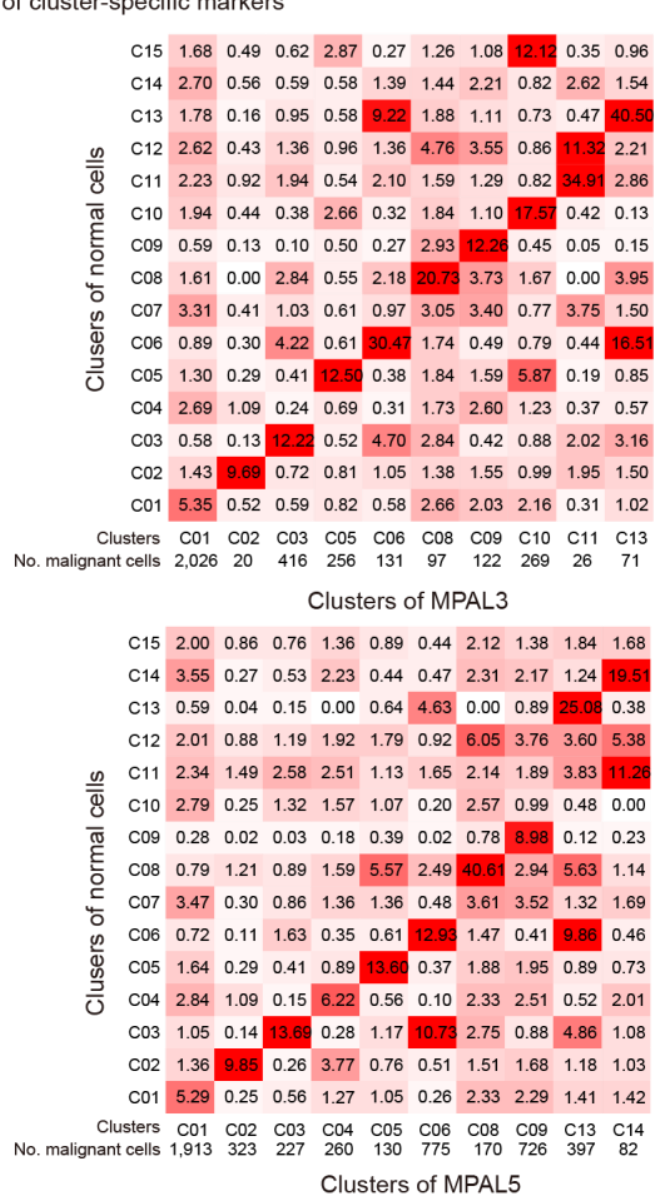

\begin{tabular}{llll|l|l|llllll} 
C15 & 1.68 & 0.49 & 0.62 & 2.87 & 0.27 & 1.26 & 1.08 & 12.12 & 0.35 & 0.96
\end{tabular} $\begin{array}{lllllllllllll}\text { C14 } & 2.70 & 0.56 & 0.59 & 0.58 & 1.39 & 1.44 & 2.21 & 0.82 & 2.62 & 1.54\end{array}$ $\begin{array}{llllllllllll}1.78 & 0.16 & 0.95 & 0.58 & 9.22 & 1.88 & 1.11 & 0.73 & 0.47 & 40.5\end{array}$ $\begin{array}{lllllllllllll}C & 11 & 2.23 & 0.92 & 1.94 & 0.54 & 2.10 & 1.59 & 1.29 & 0.82 & 34.91 & 2.86\end{array}$ $\begin{array}{llllllllllllll}\text { C10 } & 1.94 & 0.44 & 0.38 & 2.66 & 0.32 & 1.84 & 1.10 & 17.57 & 0.42 & 0.13\end{array}$ $\begin{array}{lllllllllllll}\operatorname{cog} & 0.59 & 0.13 & 0.10 & 0.50 & 0.27 & 2.93 & 12.26 & 0.45 & 0.05 & 0.15\end{array}$ \begin{tabular}{llll|l|l|l|l|l|l|l|l}
$\cos$ & 1.61 & 0.00 & 2.84 & 0.55 & 2.18 & 20.73 & 3.73 & 1.67 & 0.00 & 3.95
\end{tabular}

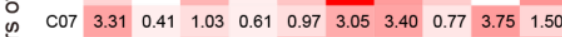
\begin{tabular}{lllllllllllllll}
\hline & C06 & 0.89 & 0.30 & 4.22 & 0.61 & 30.47 & 1.74 & 0.49 & 0.79 & 0.44 & 16.5
\end{tabular} $\begin{array}{lllllllllllllll}\operatorname{Co5} & 1.30 & 0.29 & 0.41 & 12.50 & 0.38 & 1.84 & 1.59 & 5.87 & 0.19 & 0.85\end{array}$ $\begin{array}{llll}55 & 0.99 & 1.95 & 1.50\end{array}$ $\begin{array}{llllllllll}5.35 & 0.52 & 0.59 & 0.82 & 0.58 & 2.66 & 2.03 & 2.16 & 0.31 & 1.02\end{array}$

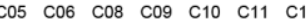
Clusters of MPAL 3 $\begin{array}{lllllllllllll}\text { C14 } & 3.55 & 0.27 & 0.53 & 2.23 & 0.44 & 0.47 & 2.31 & 2.17 & 1.24 & 19.5\end{array}$ \begin{tabular}{lllllll|l|l|l|l|l|}
$\mathrm{C} 13$ & 0.59 & 0.04 & 0.15 & 0.00 & 0.64 & 4.63 & 0.00 & 0.89 & 25.08 & 0.38
\end{tabular} $\begin{array}{llll}3.76 & 3.60 & 5.38\end{array}$

$\begin{array}{llllllllllllll}\cos & 0.28 & 0.02 & 0.03 & 0.18 & 0.39 & 0.02 & 0.78 & 8.98 & 0.12 & 0.23\end{array}$

\begin{tabular}{lllllll|l|l|l|l|l}
$\operatorname{Cos}$ & 0.79 & 1.21 & 0.89 & 1.59 & 5.57 & 2.49 & 40.61 & 2.94 & 5.63 & 1.14
\end{tabular} $\begin{array}{lllllllllllllll}\mathrm{C} 07 & 3.47 & 0.30 & 0.86 & 1.36 & 1.36 & 0.48 & 3.61 & 3.52 & 1.32 & 1.69\end{array}$ $\begin{array}{llllllllllllll}\cos & 0.72 & 0.11 & 1.63 & 0.35 & 0.61 & 12.93 & 1.47 & 0.41 & 9.86 & 0.46\end{array}$ $\begin{array}{llllllllllll}\operatorname{C0} 5 & 1.64 & 0.29 & 0.41 & 0.89 & 13.60 & 0.37 & 1.88 & 1.95 & 0.89 & 0.73\end{array}$ $\begin{array}{llllllllllllll}\text { C04 } & 2.84 & 1.09 & 0.15 & 6.22 & 0.56 & 0.10 & 2.33 & 2.51 & 0.52 & 2.01\end{array}$ $\begin{array}{lllllllllllll} & 1.05 & 0.14 & 13.69 & 0.28 & 1.17 & 1073 & 2.75 & 0.88 & 4.86 & 1.08\end{array}$ $\begin{array}{llllllllllll}\text { C02 } & 1.36 & 9.85 & 0.26 & 3.77 & 0.76 & 0.51 & 1.51 & 1.68 & 1.18 & 1.03\end{array}$ $\begin{array}{llllllllll}5.29 & 0.25 & 0.56 & 1.27 & 1.05 & 0.26 & 2.33 & 2.29 & 1.41 & 1.42\end{array}$ Clusters of MPAL5

513 Figure S6 Supplementary figures for Fig. 3. (a) The aggregated ATAC-seq profiles of normal cells (left) and bulk samples (right) on chromatin marker regions, showing biological identities of each joint cluster. (b) The fold change of enrichment between markers detected from normal cells and malignant cells. Number of malignant cells in each cluster is shown on the bottom. Clusters with fewer than 20 cells are not shown. 

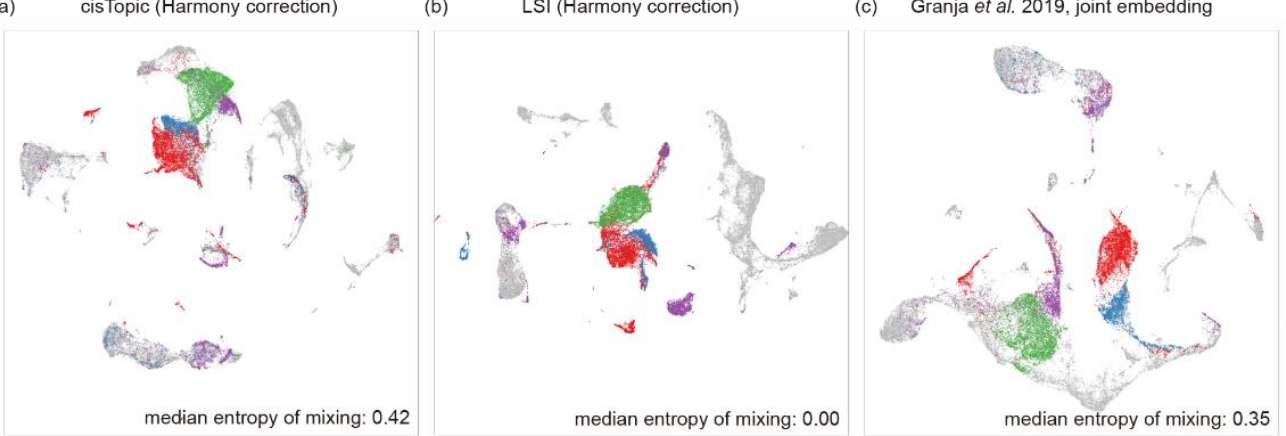

+ MPAL1

+ MPAL3

+ MPAL4

+ MPAL5

+ Normal cells

Figure S7 Benchmarking of other methods on Leukemia data. (a) CisTopic followed by Harmony

522 correction. (b) LSI followed by Harmony correction. (c) Granja et al.'s method with joint embedding. 
bioRxiv preprint doi: https://doi.org/10.1101/2020.02 13.947242; this version posted January 3, 2021. The copyright holder for this preprint (which was not certified by peer review) is the author/funder, who has granted bioRxiv a license to display the preprint in perpetuity. It is made available under aCC-BY-NC-ND 4.0 International license.

MPAL1

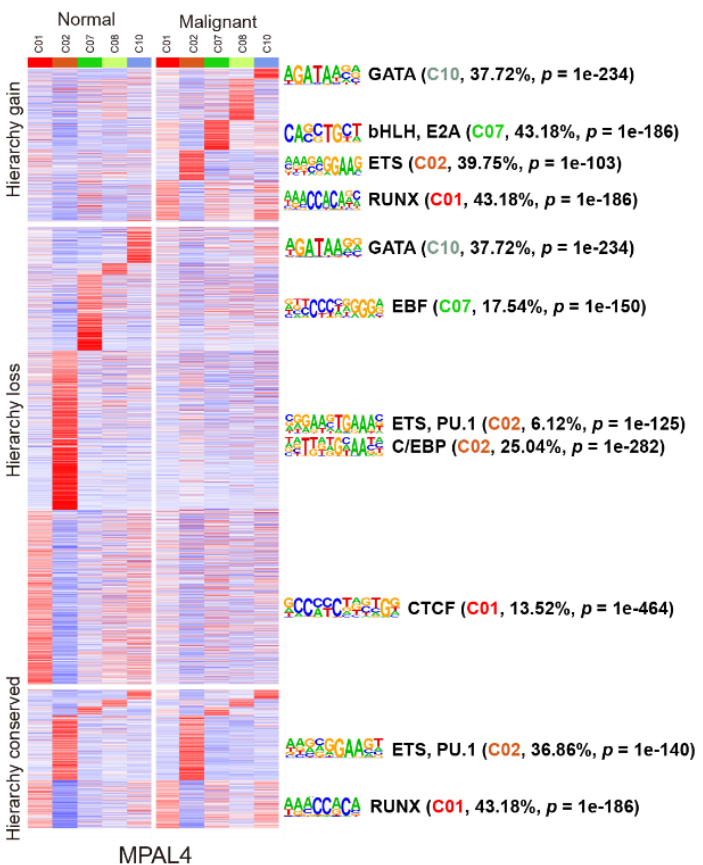

MPAL4

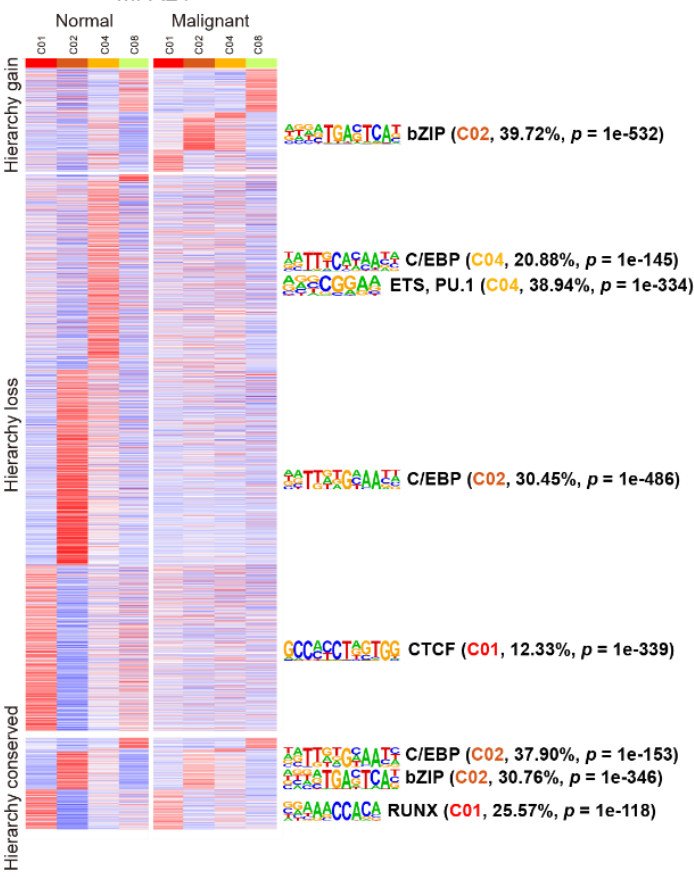

MPAL3

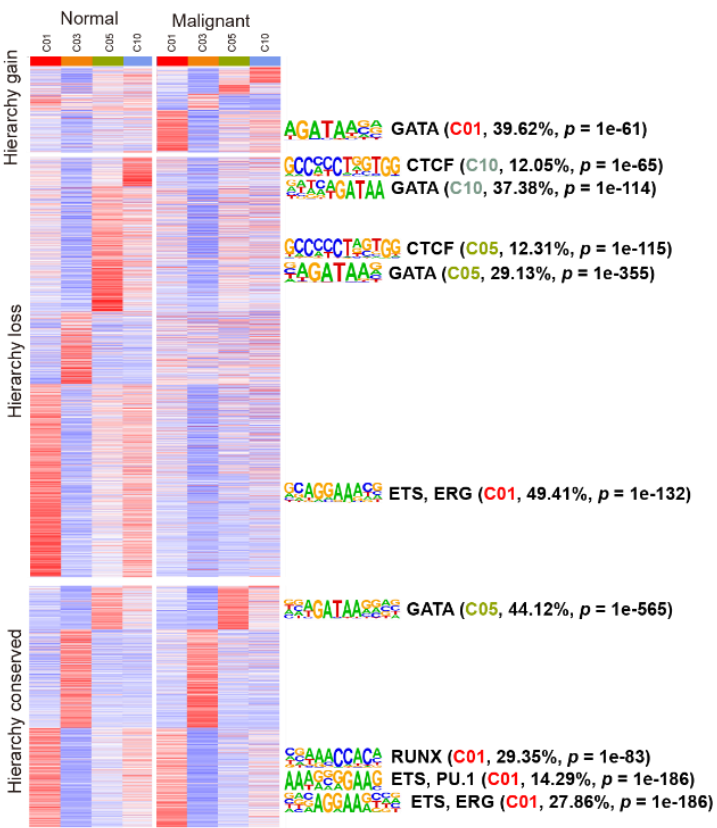

MPAL5

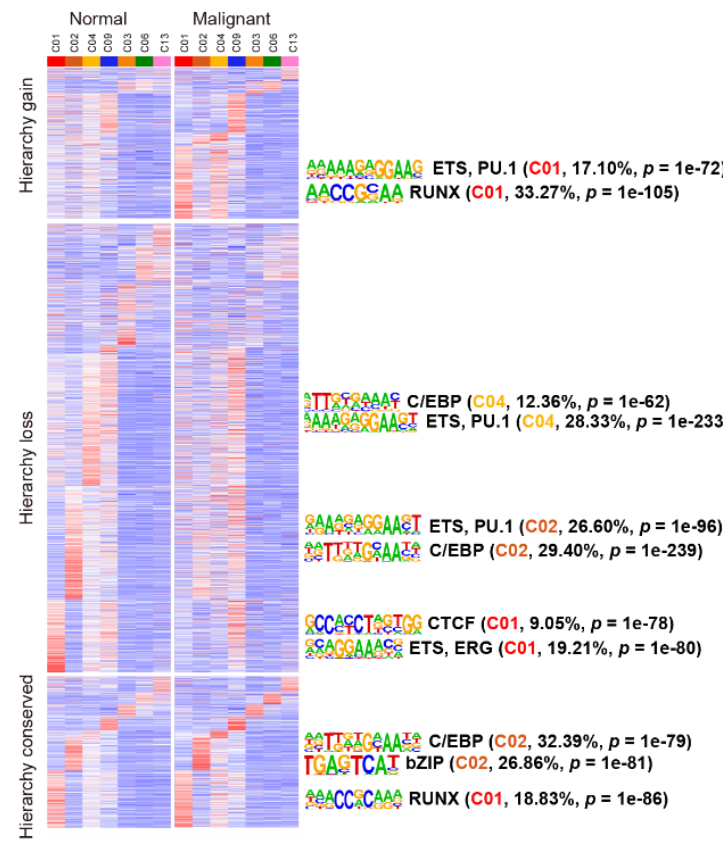

Figure S8 Aggregated ATAC-seq profiles of normal and major malignant clusters on hierarchy conserved, hierarchy loss and hierarchy gain peaks. The enriched motifs with frequency of appearance and $p$-value in each set of peaks are show in right panels. 
(a)

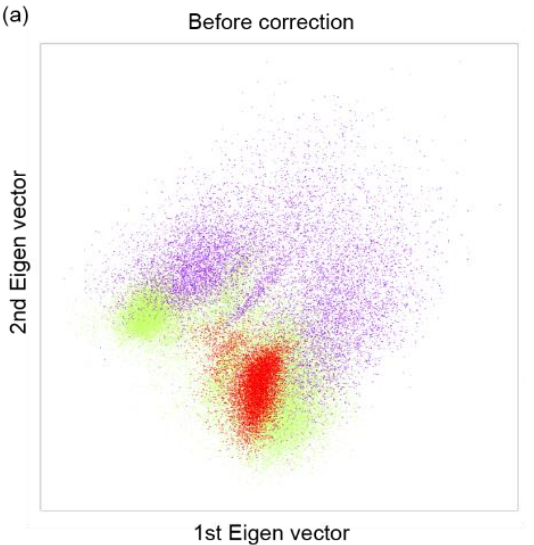

(b)

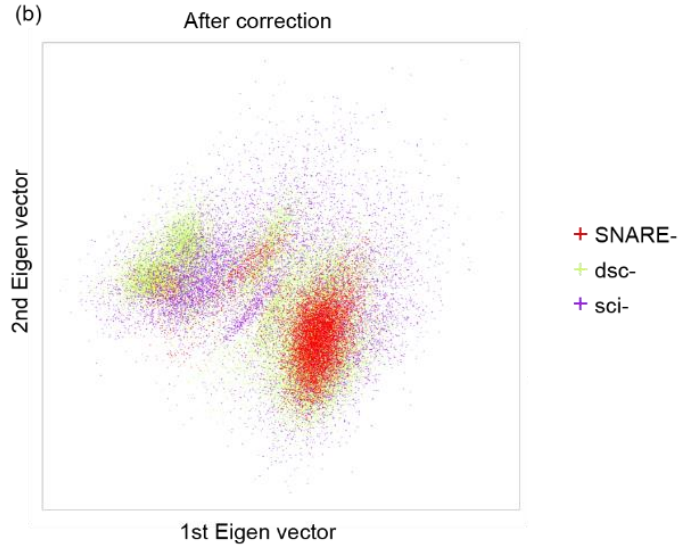

(c)

$a$ is $b$ 's true neighbor

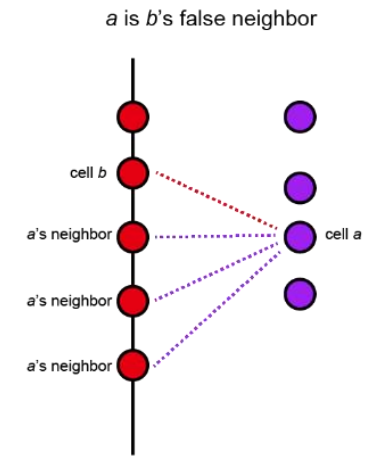

cells of dataset $A$

cells of dataset $B$

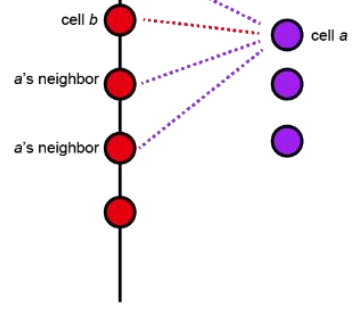

."..." $k_{1}$ neighbors

....... $k_{2}$ neighbors

__ guilding feature

(d)

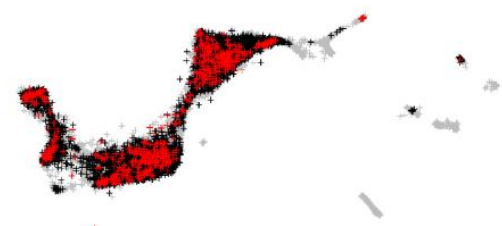

+ anchor cells (SNARE-)

+ non-anchor cells (SNARE-)

+ sci- \& dsc-

Figure S9 Algorithm details of epiConv batch correction. $(\mathbf{a}, \mathbf{b})$ The $1^{\text {st }}$ and $2^{\text {nd }}$ Eigen vectors before (a) and after (b) correction of adult mouse brain data. (c) Schematic view of removing false neighbors. (d) The distribution of anchor cells from co-assay dataset of adult mouse brain data. 
bioRxiv preprint doi: https://doi.org/10.1101/2020 02.13 .947242 ; this version posted January 3, 2021. The copyright holder for this preprint (which was not certified by peer review) is the author/funder, who has granted bioRxiv a license to display the preprint in perpetuity. It is made available under aCC-BY-NC-ND 4.0 International license.

534 Table S1 Published data used in this study with running time and memory requirements

\begin{tabular}{|c|c|c|c|c|c|}
\hline dataset & $\begin{array}{l}\text { No. cells } \\
\text { after QC }\end{array}$ & $\begin{array}{l}\text { Running time } \\
\text { (calculate similarity) }\end{array}$ & $\begin{array}{l}\text { Running time } \\
\text { (batch correction) }\end{array}$ & $\begin{array}{l}\text { Memory } \\
\text { requirement }\end{array}$ & Source \\
\hline PBMCs (Fig. 1c) & 41,909 & 5.5 hours & 0.8 hour & $<128 \mathrm{~GB}$ & $\begin{array}{l}\text { GSE129785 } \\
\text { GSE123581 }\end{array}$ \\
\hline $\begin{array}{l}\text { Adult mouse } \\
\text { brain }\end{array}$ & 53,762 & 7 hours & 2 hours & $<256 \mathrm{~GB}$ & $\begin{array}{l}\text { GSE126074 } \\
\text { GSE123581 } \\
\text { http://atlas.gs.washington.edu/ } \\
\text { mouse-atac }\end{array}$ \\
\hline $\begin{array}{l}\text { Developmental } \\
\text { mouse brain }\end{array}$ & 17,613 & 1 hour & 0.5 hour & $<32 \mathrm{~GB}$ & $\begin{array}{l}\text { GSE126074 } \\
\text { https://support.10xgenomics.co } \\
\text { m/single-cell-atac/datasets }\end{array}$ \\
\hline Leukemia & 46,510 & 6 hours & 2.1 hours & $<128 \mathrm{~GB}$ & GSE139369 \\
\hline $\begin{array}{l}5,000 \text { PBMCs } \\
\text { (Fig. S1b) }\end{array}$ & 3,936 & $<5$ minutes & & $<8 \mathrm{~GB}$ & $\begin{array}{l}\text { https://support.10xgenomics.co } \\
\mathrm{m} / \text { single-cell-atac/datasets }\end{array}$ \\
\hline Cell lines & 1,174 & $<5$ minutes & & $<2 \mathrm{~GB}$ & GSE65360 \\
\hline
\end{tabular}




\section{Supplementary Note 1}

Here we describe a simple method to calculate the similarities between single cells of ATAC-seq

data. First, assume that we have two distributions, $p_{X}$ and $p_{Y}$, where cells sample their insertion events from (the chromatin states of two types of cells). If we sample one insertion from $p_{x}$ and one insertion from $p_{y}$, we have a new random variable that is 1 if two insertions occur in the same peak or 0 otherwise. Given random binary vectors $X$ with $m$ non-zero elements sampled from $p_{X}$ and $Y$ with $n$ non-zero elements sampled from $p_{Y}$, the dot product of $X$ and $Y$ can be considered as the sum of $m \times n$ variables and is determined by $p_{X}$ and $p_{Y}$ if $X$ and $Y$ are sparse. Thus, the $X \cdot Y /(m \cdot n)$ can be used to measure the similarity between $X$ and $Y$. However, the value of $X \cdot Y$ is mainly affected by the peaks that have higher frequency. To balance the contribution of peaks with high frequency and low frequency, we weight each peak by $w=$ $\log _{10}(1+$ inverse frequency of peak $)$ following LSI, where peaks with higher frequency have lower weight. The observations from real data also support our assumption that the $X \cdot Y$ can be modeled as $m \times n$ random variables (Fig. a below).

In order to reduce the noise of similarity measurement, we adopt a bootstrap strategy. In ith bootstrap, we calculate $\log _{10}\left(X_{i} \cdot Y_{i}\right)-\log _{10} m-\log _{10} n$, where we randomly sample some peaks from $X$ and $Y$ to generate $X_{i}$ and $Y_{i}$ (we sampled 20\% peaks in each bootstrap and performed 15 bootstraps in this study), and use the mean of $\log _{10}\left(X_{i} \cdot Y_{i}\right)-\log _{10} m-\log _{10} n$ across bootstraps as the similarity between cells.

Even after normalization by $m \times n$, there are still some dependencies between the similarities and the library sizes. First, similarities between cells with small library sizes tends to have large variations (Fig. $\mathbf{b}$ below), which is consistent with our initial assumption as the mean value of 
bioRxiv preprint doi: https://doi. org/10.1101/2020.02 13.947242; this version posted January 3, 2021. The copyright holder for this preprint (which was not certified by peer review) is the author/funder, who has granted bioRxiv a license to display the preprint in perpetuity. It is made available under aCC-BY-NC-ND 4.0 International license.

558 fewer random variable has larger variation. Second, there are weak negative correlation between

559 the similarity and library size (Fig. $\mathbf{b}$ below, coefficient $\approx-0.05$ in most cases), as the $m \times n$

560 variables are not independent. We perform linear regression on log transformed similarities and

$561 \log _{10} m+\log _{10} n$. The regression residuals are further divided by the corresponding standard

562 deviations, calculated by data points with similar $\log _{10} m+\log _{10} n$. The regression residuals

563 after variance stabilization are used as the similarity scores between two cells (Fig. c below).

(a)

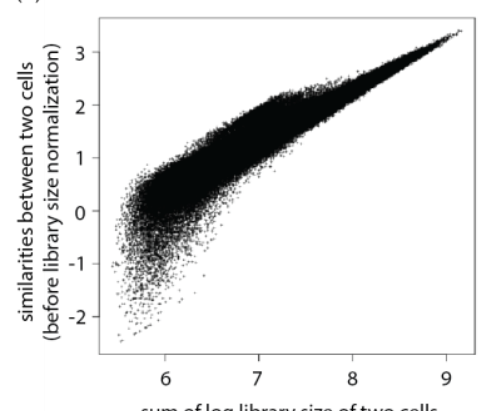

565

566 (b)

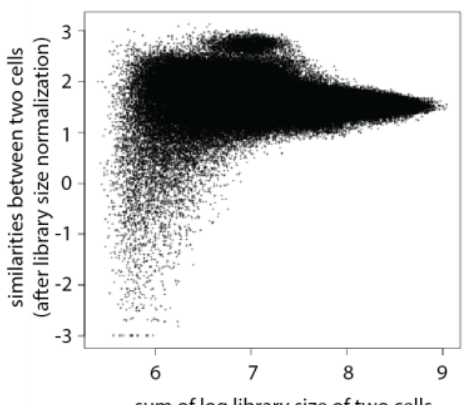

sum of log library size of two cells (c)

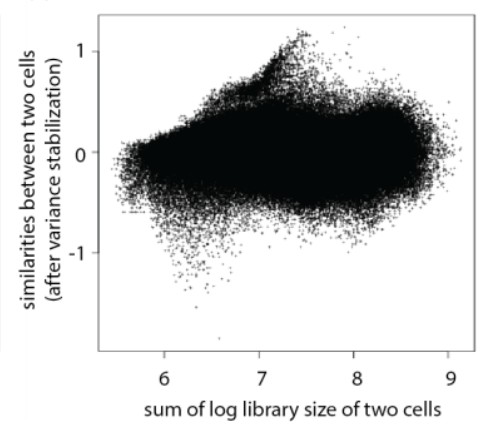

